\title{
Optimal treatments for hepato-pancreato-biliary trauma in severely injured patients: a narrative scoping review
}

\author{
Lucas Streith, MD* \\ Jenna Silverberg, BSc* \\ Andrew W. Kirkpatrick, MD \\ S. Morad Hameed, MD \\ Oliver F. Bathe, MD \\ Chad G. Ball, MD
}

This study was presented at Canadian Surgery Forum in Montreal, Quebec, Canada on September 5, 2019.

${ }^{*}$ These authors contributed equally to this work.

Accepted Dec. 18, 2019

\section{Correspondence to: \\ C. Ball \\ 1403-29 St NW \\ Foothills Medical Centre \\ Calgary AB T2N 5A1 \\ ball.chad@gmail.com}

DOI: $10.1503 /$ cjs.013919

\section{SUMMARY}

Hepato-pancreato-biliary (HPB) injuries can be extremely challenging to manage. This scoping review (8438 citations) offers a number of recommendations. If diagnosis and therapy are rapid, patients with major hepatic injuries who present in physiologic extremis have high survival rates despite prolonged hospital stays. Nonoperative management of major liver injuries, as diagnosed using computed tomography, is typically successful. Adjuncts (e.g., angioembolization, laparoscopic washouts, biliary stents) are essential in managing high-grade injuries. Injury to the extrahepatic biliary tree is rare. Cholecystectomy is indicated for all gallbladder trauma. Full-thickness common bile duct injuries require a hepaticojejunostomy, although damage control remains closed suction drainage. Injuries to the pancreatic head often involve concurrent trauma to regional vasculature. Damage control necessitates drainage after stopping hemorrhage. Injury to the left pancreas commonly requires a distal pancreatectomy. Outcomes for high-grade pancreatic and liver injuries are improved by involving an HPB team. Complications are multidisciplinary and should be managed without delay.

njuries to the liver, extrahepatic biliary tree and pancreas (i.e., the "surgical soul") are often deadly and always challenging. These scenarios will engage all of your senses, test your skills, and demand great teamwork from you and your colleagues. In an effort to offer a continuing medical education review of this topic that consolidates the current published advice on treating hepato-pancreato-biliary (HPB) injuries, we performed a narrative scoping analysis of the literature.

Our literature search protocol was developed according to recommendations for designing scoping reviews. ${ }^{1}$ Three independent investigators searched MEDLINE, EMBASE, PubMed, Web of Science, Scopus and the Cochrane Library (English language) from their inception dates through to May 1, 2019, without restrictions. Disagreements were resolved by consensus. Literature on bile duct injuries during elective/ emergency cholecystectomy and/or pediatric trauma was excluded. The grey literature (conference abstracts (1988-2019) of the American Association for the Surgery of Trauma, Australasian Trauma Society, Eastern Association for the Surgery of Trauma, Trauma Association of Canada, Western Trauma Association, and 24 textbook chapters) was also searched for discussions not reported in peer-reviewed articles.

\section{General Results}

Of the 8438 citations identified, 183 (11.6\%) unique articles focused on HPB trauma were included in the scoping review. Agreement among investigators regarding selection of abstracts for full-text review (k-statistic $0.91,95 \%$ confidence interval [CI] 0.88-0.94) and inclusion of articles in the scoping review (k-statistic $0.90,95 \%$ CI 0.89-0.92) was excellent. 
These HPB-specific trauma articles focused on the liver (109), pancreas (102), duodenum (25) and extrahepatic biliary tree (5). Most articles $(86.3 \%)$ were retrospective series with a median of 107 patients. Only $9.3 \%$ were prospective. Most (74.8\%) studies were conducted at level 1 trauma centres. Although many articles focused on a mix of blunt and penetrating mechanisms (41.5\%), some were specific to blunt $(32.2 \%)$ or penetrating $(11.5 \%)$ injuries. Articles were commonly American (50.8\%) or South African (11.5\%).

\section{Liver}

Although textbooks and articles have traditionally been filled with complex hierarchies of operative manoeuvres for treating hepatic trauma, it is clear that the vast majority of liver injuries (94\% in the literature) are now treated nonoperatively. This process involves diagnosis with cross-sectional imaging (computed tomography $[\mathrm{CT}]$ ), followed by serial clinical and laboratory (hemoglobin, white blood cell count [WBC], liver function tests/enzymes) monitoring. This algorithm allows the clinician to reliably predict and treat both the initial injury and potential complications. Any patient who presents with hypotension and/or peritonitis requires emergent operative therapy.

The dominant challenge with high-grade hepatic trauma is management of hemodynamically unstable patients with ongoing hemorrhage. These patients often present in physiologic extremis and require damage control (DC) resuscitation techniques. ${ }^{2-5}$ Early recognition of their critical condition, as well as immediate hemorrhage control, is essential for survival. Patients with major injury caused by blunt trauma or right upper quadrant penetrating mechanisms should undergo an immediate ultrasonography (extended focused assessment with sonography in trauma) examination upon arrival to confirm the presence of significant intraperitoneal fluid. This exam is repeatable and can be used to re-evaluate patients in urban centres when presentation immediately follows injury. Massive transfusion protocols, as part of a DC resuscitation, must be initiated early during the patient's assessment. If the patient's hemodynamics stabilize rapidly, they should undergo an emergency CT scan of the torso (blunt and gunshot). In the context of right upper quadrant stab wounds, CT may also be helpful (although debated) to rule out hepatic injury and/or associated diaphragm injuries. If the patients remain clinically unstable, however, they should be transferred to the operating theatre without delay. Hemorrhage control is the dominant priority in these patients. Collateral issues such as optimal intravenous access, additional imaging (brain, spine, bones), and fracture fixation remain secondary priorities. In summary,
- in hemodynamically stable patients without CT evidence of a hepatic arterial blush or other reasons to proceed to the operating theatre, close observation is warranted;

- in hemodynamically stable patients with a hepatic arterial extravasation, immediate transfer to the interventional angiography suite (or hybrid operating theatre) is mandated for hepatic angiography and/or portography with selective embolization (autologous clot or absorbable medium is preferred);

- in patients with persistent hemodynamic instability, immediate transfer to the operating theatre for laparotomy is essential. Delays will lead to loss of life.

Operative tips in the literature for treating liver trauma are multifold. The peritoneal cavity should be packed with laparotomy sponges for patients with blunt liver injuries. In those with lacerations to the right lobe, the falciform ligament may be left intact to provide a medial wall against which to improve packing pressure. The goal of perihepatic packing is to reconstitute the liver back into its normal morphology. The right upper quadrant (and midline vascular structures) should be evaluated before any intraperitoneal packing for penetrating injuries. If hemorrhage continues despite packing, an early Pringle manoeuvre (porta hepatis occlusion with a vascular clamp) is mandated. This is both diagnostic and potentially therapeutic. If bleeding persists despite application of a Pringle, a retrohepatic inferior vena cava (IVC) or hepatic venous injury is likely. In this scenario, call for senior assistance, mobilize the right lobe and suture the IVC/hepatic veins with 4-0 (SH) or 5-0 prolene (RB-1) (assuming a replaced left hepatic artery is not the source of inflow occlusion failure). Critically injured patients in physiologic extremis do not tolerate extended Pringle manoeuvres (> 40 minutes) to the same extent as those undergoing elective hepatic resection. If hemorrhage responds to perihepatic packing $(85 \%-99 \%$ of cases in the literature), but restarts when unpacked, it should be repacked and transferred to the intensive care unit with an open abdomen once DC of concurrent injuries is complete. Covering the liver with a plastic layer of sterile $x$-ray cassette material to avoid capsular trauma upon eventual unpacking is helpful. Topical hemostatic agents can also be beneficial. Patients with ongoing hemorrhage and/or ruptured retrohepatic hematomas may also require total vascular exclusion (TVE) of the liver (complete occlusion of the infrahepatic IVC, suprahepatic IVC, porta hepatis [Pringle]). If TVE is pursued without concurrent aortic clamping, the patient may arrest due to a lack of coronary perfusion. As a result, the surgeon must be ready to rapidly apply this occlusion technique if it is not performed prophylactically. Suprahepatic IVC control can occur within the abdomen in patients with a normal length of IVC 
immediately inferior to the diaphragm. Alternatively, the $2 \mathrm{~cm}$ length of IVC within the pericardium (i.e., as it enters the heart) is easily accessible by opening the pericardial sac after division of the diaphragm (or within the thorax if a thoracotomy is already completed). Veno-veno bypass is a theoretical option, but rarely used because of a lack of transplant training by most trauma surgeons.

For central hepatic gunshot wounds or deep central lacerations where exposure is difficult, ongoing hemorrhage can be stopped with balloon occlusion. ${ }^{6} \mathrm{~A}$ Blakemore esophageal balloon (or red rubber catheter with overlying Penrose drain and 2 silk ties) is exceptional at stopping bleeding deep within hepatic injury tracts (including retrohepatic IVC). Catheters should be deflated approximately 72 hours after the initial placement. If hemorrhage continues, they should be left in place for 2-3 additional days. Adjunctive angiography addressing concurrent arterial hemorrhage is also advised. Although unusual, penetrating injuries to the hepatic artery may undergo repair or require ligation (if the portal vein is intact). Portal vein injuries should ideally be repaired with $5-0 / 6-0$ prolene once control above and below is obtained. Alternate DC options include temporary intravascular shunt (TIVS) with a small chest tube conduit, or ligation (if hepatic artery is intact). Return to the operating suite in patients with packed open abdomens should occur within 48-72 hours (once hypothermia, coagulopathy and acidosis are corrected). If an atriocaval shunt is contemplated, 2 experienced surgical teams (1 chest/ 1 abdomen) are essential. Pursuit of this shunt must occur early in the exploration process as they rarely result in patient salvage.

Morbidity following hepatic trauma includes early (bleeding, abdominal compartment syndrome) and late (infected hematoma, bile leak, liver failure, ileus) complications. Laparoscopic washout with copious irrigation and insertion of closed suction drainage catheter(s) is helpful in patients who have large collections of infected hematoma or biloma. This is not uncommon with high-grade hepatic injuries $(8 \%-10 \%$ risk of biloma in grade III-V injuries). If a bile leak persists (> 1-2 weeks), placement of a biliary stent via endoscopic retrograde cholangiopancreatography (ERCP) often encourages closure. As a result, successful "nonoperative" therapy of high-grade liver injuries requires access to percutaneous drainage, laparoscopy, ERCP and/or angiography.

Despite a known 4-8 week hypertrophy response following elective resection, the time to complete organ healing after hepatic trauma is unclear. Unlike splenic lacerations, planned surveillance imaging is not required for major liver injuries. Repeat imaging should be based on laboratory or patient deterioration.

\section{Extrahepatic biliary tree}

Injuries to the extrahepatic biliary tree (common bile duct and gallbladder) are very uncommon. Diagnosis typically occurs within the operating theatre at the time of urgent repair for concurrent injuries. Trauma to the biliary tree is suspected upon identification of bile within the peritoneal cavity. If the diagnosis is delayed, a biloma will form and the patient may report nausea, discomfort and an ileus. Their WBC and bilirubin levels may also increase. These patients require identification of the biloma (ultrasonography or CT), in addition to subsequent percutaneous drainage and cholangiography. In scenarios of very small partialthickness common bile duct injuries, placement of a biliary stent via ERCP may be sufficient. With a severe injury, however, immediate control of sepsis and subsequent appropriately timed exploration is warranted. In this setting, complete cholangiography and consultation with an experienced HPB surgery team approach is essential before exploration.

Any injury to the gallbladder is an absolute indication for cholecystectomy. ${ }^{7}$ Although primary repair is occasionally described in large series, it is almost never recommended. A full-thickness common bile duct injury requires a Roux-en-Y hepaticojejunostomy to minimize the long-term risk of anastomotic strictures (an end-toside hepaticoduodenostomy is also reasonable in the absence of tension). In the setting of small, partial thickness sharp (i.e., nonblunt/nongunshot) injuries, primary repair with $\mathrm{T}$-tube drainage remains a viable option. Damage control for any extensive biliary injury consists of control of the bile leakage via closed suction drainage.

The dominant long-term potential complication after hepaticojejunostomy remains anastomotic stricture. This is particularly true in young patients with a long life expectancy. It mandates a detailed discussion with the patient before discharge (i.e., risks and symptoms of potential stenosis [cholangitis, jaundice]). These patients are often amenable to dilations of their anastomoses with either an endoscopic or percutaneous approach.

\section{Pancreas}

Trauma to the pancreas can be challenging for even the most experienced surgeon. ${ }^{8}$ It is divided into injuries to the left (body/tail) and right (head) of the portal vein..$^{9,10}$ Injuries to the right pancreas also place adjacent structures (portal vein, IVC, aorta, duodenum, bile duct) at risk. Absolute indications for operative exploration of the pancreas include persistent hypotension, diffuse peritonitis, and full-thickness pancreas disruption on initial CT.

In patients undergoing a laparotomy, all pancreatic hematomas should be opened and explored to define the 
integrity of the pancreatic capsule. All segments of the pancreas can be directly inspected by using standard exposure manoeuvres (Kocher, division of ligament of Treitz, entry into the lesser sac, medial mobilization of the pancreas tail/spleen). If a disruption in the pancreatic capsule is noted (Grade I or II), a closed suction drain should be placed adjacent to the injury. If a pancreatic laceration is noted in the setting of an intact main pancreatic duct, an omental plug can be combined with closed suction drainage. In general, injuries with disruption of the main pancreatic duct (Grade III) at any location to the left of the superior mesenteric vein/portal vein should undergo a distal pancreatectomy. In patients with multiple critical injuries, this procedure can be delayed for a few hours while other priorities are addressed. Across a truly disrupted main pancreatic duct, ERCP-based pancreatic duct stenting fails almost uniformly. Similarly, a Roux-en-Y pancreaticojejunostomy to the pancreatic segment distal to the transaction is fraught with complications because of the soft texture of an otherwise normal gland.

Defining the integrity of the main pancreatic duct can be challenging. Ultrasonography has become incredibly valuable for HPB surgeons. In the absence of this skill, cholecystocholangiography (methylene blue mixed with radio-opaque contrast) remains the best alternative (i.e., poor test performance of CT).

Substantial injuries to the right pancreas (head) with main duct disruption typically require a pancreatoduodenectomy. In the context of DC resuscitation owing to massive hemorrhage, the initial surgery does not typically include either a completed resection or reconstruction. As a result, the "trauma Whipple" is often performed in 2 stages. The second procedure (resection completion/final reconstruction) should be pursued within 24-48 hours. If re-exploration is delayed, the viscera often become so edematous that reconstruction is extremely challenging because of limited mobility of the pancreato-biliary limb, as well as edematous pancreatic tissue. It should also be noted that most combined pancreatoduodenal injuries do not require a pancreatoduodenectomy. In these patients (with ampullary, common bile and pancreatic duct integrity), procedures such as primary repair or pyloric exclusion with gastrojejunostomy may be superior options. The "bail-out" manoeuvre for pancreatic head trauma remains closed suction drainage with early re-exploration in the company of an experienced colleague.

Regardless of the technique, up to $25 \%$ of distal pancreatectomies generate a pancreatic leak and subsequent fistula. This may be higher in the setting of trauma due to concurrent injuries and physiologic stress. Soft texture, small duct, contaminated field, and bruised gland makes pancreatic surgery both challenging and fraught with complications in the injured patient. Wrapping the pancreas stump with viable omentum can be helpful.
Octreotide has not been reliably shown to reduce the time to closure of pancreatic fistulas in injured patients. In addition to these early complications, delayed endocrine insufficiency is a concern (if $>60 \%$ of the pancreas is removed in young trauma patients, half will eventually display glucose intolerance, and up to $25 \%$ will develop diabetes).

\section{Conclusion}

Timely management of complex hepato-pancreatobiliary trauma requires strong clinical pattern recognition, multidisciplinary team collaboration, and longterm patient follow-up. Management of these injuries continues to evolve and become more nuanced with both improved techniques and an enhanced understanding of underlying HPB diseases.

Affiliations: From the Department of Surgery, University of Calgary, Calgary, Alta. (Streith, Silverberg, Kirkpatrick, Bathe, Ball); and the Department of Surgery, University of British Columbia, Vancouver, BC (Hameed).

Competing interests: C. Ball is co-editor in chief of $C F S$; he was not involved in the review or decision to accept this manuscript for publication. No other competing interests were declared.

Contributors: All authors contributed substantially to the conception, writing and revision of this article and approved the final version for publication.

\section{References}

1. Daudt HM, van Mossel C, Scott SJ. Enhancing the scoping study methodology: a large, inter-professional team's experience with Arksey and O'Malley's framework. BMC Med Res Methodol 2013;13:48.

2. Roberts DJ, Ball CG, Feliciano DV, et al. History of the innovations of damage control for management of trauma patients: 1902-2016. Ann Surg 2017;265: 1034-44.

3. Calne RY, McMaster P, Pentlow BD. The treatment of major liver trauma by primary packing with transfer of the patient for definitive treatment. Br 7 Surg 1979;66:338-9.

4. Feliciano DV, Mattox KL, Burch JM, et al. Packing for control of hepatic hemorrhage. 7 Trauma 1986;26:738-43.

5. Ball CG. Damage control resuscitation: history, theory and technique. Can 7 Surg 2014;57:55-60.

6. Ball CG, Wyrzykowski AD, Nicholas JM, et al. A decade's experience with balloon catheter tamponade for the emergency control of hemorrhage. 7 Trauma 2011;70:330-3.

7. Ball CG, Dixon E, Kirkpatrick AW, et al. A decade of experience with injuries to the gallbladder. 7 Trauma Manag Outcomes 2010;4:3.

8. Asensio JA, Petrone P, Oluwaseye AO, et al. Laparotomy: the conquering of the abdomen and the historical journey of pancreatic and duodenal injuries. 7 Trauma Acute Care Surg 2016; 80:1023-31

9. Biffl WL, Moore EE, Croce M, et al. Western trauma association critical decisions in trauma: management of pancreas injuries. 7 Trauma Acute Care Surg 2013;75:941-6.

10. Ho VP, Patel NJ, Bokhari F, et al. Management of adult pancreatic injuries: A practice management guideline from the Eastern Association for the Surgery of Trauma. 7 Trauma Acute Care Surg 2017; 82:185-99. 